\title{
Delimitación taxonómica de Ceiba chodatily C. speciosa (Malvaceae, BombacoideAe) en diferentes estadios fenológicos
}

\author{
EVANGELINA C. LOZANO ${ }^{1}$ y MARÍA A. ZAPATER ${ }^{1}$
}

\begin{abstract}
Summary: Taxonomic delimitation of Ceiba chodatii and C. speciosa (Malvaceae, Bombacoideae) in different phenological stages. Three species of Ceiba (Malvaceae, Bombacoideae) are cited for Argentina: C. chodatii, C. speciosa and C. pubiflora, differentiated by a few characters. The first two species are clearly distinguished in flowering stage, being difficult to identify them in other stages. The objective of this investigation is to check comparatively $C$. chodatii and $C$. speciosa in the search of greater characters that contribute to its delimitation. Native specimens of $C$. chodatii were studied in the Selva pedemontana and in Chaco serrano, also of $C$. speciosa cultivated in Salta and from herbaria. The vegetative and reproductive characters were studied with binocular loupe and MEB. A germination and seedling development test was carried out. The results show that the leafs from seedling to adult are differentiated in nerves and serrated of the margin. The differential characters in flower are: the size of the lobes in the staminodium column and the pubescence, the size of the anthers and collar, the total or partial union of the filaments, the shape, size and pubescence of the stigma and the type of basal pubescence of style. The fruits are differentiated by the thickness of the valves and the seeds by pubescence.
\end{abstract}

Key words: Ceiba, delimitation, taxonomy, phenological stages.

\begin{abstract}
Resumen: Para Argentina se citan tres especies de Ceiba (Malvaceae, Bombacoideae): C. chodatii, C. speciosa y C. pubiflora, diferenciadas por pocos caracteres. Las dos primeras especies se distinguen claramente en etapa de floración, siendo complicada su identificación en otros estadios. El objetivo de esta investigación es revisar comparativamente a $C$. chodatii y $C$. speciosa en la búsqueda de mayores caracteres que aporten a su delimitación. Se estudiaron ejemplares nativos de $C$. chodatii en la Selva pedemontana y en Chaco serrano, también de C. speciosa cultivados en Salta y provenientes de herbarios. Se estudiaron todos los caracteres vegetativos y reproductivos, con lupa binocular y MEB. Se realizó un ensayo de germinación y desarrollo de plántulas. Los resultados muestran que las hojas desde plántula hasta adulto se diferencian en nerviación y aserrado del margen. Los caracteres diferenciales en flor son: el tamaño de los lóbulos en la columna de estaminodios y la pubescencia, el tamaño de las anteras y collar, la concrescencia total o parcial de los filamentos estaminales, la forma, tamaño y pubescencia del estigma y el tipo de pubescencia basal del estilo. Los frutos se diferencian por el espesor de las valvas y las semillas por pubescencia.
\end{abstract}

Palabras clave: Ceiba, delimitación, taxonomía, estadios fenológicos.

\section{INTRODUCCIÓN}

Recientes estudios filogenéticos basados en datos morfológicos, anatómicos, moleculares y biogeográficos muestran que las Bombacaceae como tradicionalmente se las circunscribía no constituyen un grupo monofilético. Las Bombacaceae no son reconocidas por el Angiosperm Phylogeny Group

\footnotetext{
${ }^{1}$ Facultad de Ciencias Naturales, Universidad Nacional de Salta, Avda. Bolivia 5150, (4400) Salta, Argentina. E-mail: evangelozano@gmail.com
}

(APG IV, 2016) con el rango de familia; se las trata como subfamilia Bombacoideae dentro de la familia Malvaceae (s.1.), clado que incluye miembros de cuatro familias previamente reconocidas Malvaceae (s.s), Bombacaceae, Tiliaceae y Sterculiaceae. Las subfamilias Bombacoideae y Malvoideae forman el clado Malvatheca (Baum et al., 1998) soportado por secuencias matK y ndhF (cpDNA) y las anteras altamente modificadas, bi - monotecas y poliesporangiadas. La subfamilia Bombacoideae comprende los taxa de hojas palmadas de Bombacáceas y la subfamilia Malvoideae incluye a las Malváceas (s.s.) (Baum et al., 2004). Kunth 
(1822) describió al género Chorisia, con dos especies, Ch. insignis y Ch. crispiflora, caracterizado por la fusión completa de los filamentos estaminales funcionales para dar un tubo alrededor del estilo. Ceiba Mill. fue reestablecido por Schumann (1886, 1890) que reconoció tres secciones para Ceiba y consideró que los géneros Ceiba y Chorisia tenían caracteres para mantenerse unidos; lo mismo fue propuesto posteriormente por Gibbs et al. (1988). Por ello, Ceiba insignis (Kunth) P.E. Gibbs \& Semir es sinónimo de Chorisia insignis Kunth. También el complejo Chorisia integrado por Ch. crispiflora Kunth, Ch. speciosa A. St.-Hil., Ch. ventricosa Nees \& Mart., Ch. integrifolia Ulbr. y Ch. incana A. Robyns, fue transferido por Ravenna (1998) a Ceiba, aunque diferentes estudios tratan a estos taxa como sinónimos de Ceiba insignis y consideran que son un complejo polimórfico del que podrían derivar especies o subespecies como resultado de estudios detallados. Gibbs \& Semir (2003) establecieron dos secciones. En la sección Ceiba, descripta en base al tipo C. pubiflora (A. St.-Hil.) $\mathrm{K}$. Schum, caracterizada por poseer polen peritremo esférico y apéndices estaminales vascularizados cuando presentes, está el complejo C. insignis con los siguientes taxa: C. pubiflora, C. chodatii (Hassl.) Ravenna, C. insignis, C. ventricosa, $C$. lupuna P. G. Gibbs \& Semir, C. speciosa, C. crispiflora, que presentan el tubo estaminal entero terminado en collar de anteras y solo una especie de este conjunto, C. pubiflora, tiene estambres libres. La distribución se corresponde con el Arco Pleistocénico de bosques estacionales definido por Prado \& Gibbs (1993), a excepción de dos especies con amplia distribución C. speciosa y C. lupuna que pueden encontrarse en bosques húmedos ribereños. Según estos autores, todo el agregado es interfértil, C. speciosa $\times$ C. chodatii forman híbridos fértiles algunos de los cuales están comúnmente cultivados en arbolado en Argentina; también C. pubiflora $\times C$. speciosa pueden cruzarse.

Ceiba comprende árboles grandes, a veces con tronco ventricoso, tronco y ramas generalmente con fuertes aguijones cónicos y hojas palmaticompuestas con 3-5 (-8) folíolos (Stevens et al., 2001). Tres especies se encuentran en México y Centro América y 13 especies se distribuyen en América del Sur. Para Argentina se citan tres especies: Ceiba chodatii, Ceiba speciosa y Ceiba pubiflora var. pubiflora (Zuloaga et al., 2008). C. chodatii habita en Argentina en la Selva Pedemontana y Chaco serrano de Salta, Jujuy, Tucumán, Catamarca y la zona de ecotono con el Chaco occidental; también en Chaco, Formosa y Santiago del Estero (Digilio \& Legname, 1966). C. speciosa, árbol nativo de las selvas tropicales y subtropicales de Sudamérica, habita en Argentina en Misiones, Corrientes, este de Chaco y Formosa y norte de Santa Fe (Selva Paranaense y Chaco oriental) (Erize, 1997). C. pubiflora var. pubiflora habita en Argentina en un área reducida (Misiones - Candelaria) (Anton \& Zuloaga, 2017).

Ceiba chodatii y C. speciosa son las dos especies que ofrecen dificultad para su identificación en estado vegetativo o en fructificación fuera de su hábitat natural por encontrarse muy cultivadas y tener gran afinidad morfológica; las diferencias conocidas se basan solo en caracteres florales no cuantitativos. Según Novara (1993), C. chodatii es una especie imposible de confundir con ninguna otra indígena del valle de Lerma, pero muy difícil de distinguir de C. speciosa, "samohú" o "samuhú", del noreste argentino, cuando faltan flores. Para este autor, posiblemente ambos taxones no sean más que variedades de una misma entidad. Por otra parte, Gibbs \& Semir (2003), mencionan la existencia de hibridación natural, aunque no se encontraron registros que lo confirmen en Argentina.

El objetivo del trabajo es revisar comparativamente a $C$. chodatii y $C$. speciosa en todos los estadios del desarrollo en la búsqueda de otros caracteres, principalmente vegetativos, que aporten a su identificación.

\section{Material y Método}

La metodología que se utilizó fue la tradicional para los estudios taxonómicos consistente en observaciones macroscópicas y con lupa binocular. Se consultaron los herbarios: LIL y MCNS. Se realizaron nuevas colecciones de $C$. chodatii en el área de distribución natural en Jujuy, Salta y Tucumán (Selva Pedemontana y Chaco Serrano), en diferentes estadios. También se coleccionaron ejemplares cultivados de esta especie y de $C$. speciosa. De esta última se seleccionaron ejemplares típicamente característicos por la coloración de los pétalos. Los ejemplares fueron descriptos y comparados en forma completa con mediciones de 


\section{E. C. Lozano y M. A. Zapater - Delimitación taxonómica en Ceiba}

todos los órganos y sus partes. Se coleccionaron frutos y semillas y también se produjeron plántulas para analizar morfología, germinación y desarrollo.

Al MEB se analizaron algunos órganos como base del estilo, estigma y episperma. Para ello, las flores fueron fijadas en FAA para su posterior análisis más detallado en el Microscopio Electrónico de Barrido (MEB; JEOL JSM-6480 LV) y tratadas con las técnicas convencionales propias de este tipo de estudio.

Se consultaron en JSTOR Plant Science las imágenes digitales de los ejemplares tipo depositados en G, MO y P (Thiers, 2017). Se confeccionaron claves para la identificación de las especies.

\section{Resultados}

Se analizaron características diferenciales detalladas en trabajos previos y se encontraron nuevas diferencias que se mencionan a continuación, con dos claves dicotómicas que se proponen.

\section{Porte especifico, forma del tronco}

En ejemplares maduros de $C$. chodatii el tronco se ensancha abruptamente desde la base hasta el ápice alcanzando en la parte media una gran dilatación (hasta $2 \mathrm{~m}$ diám.), lo que permite diferenciarlo de C. speciosa con tronco más estilizado por un ensanchamiento gradual y con menor dilatación en la mitad de la longitud (hasta 1 m diám.). Fig. 1, A-B.

\section{Aguijones}

En C. chodatii los aguijones del tronco son de forma conoidal con base muy ancha, dilatada y progresivamente aguzada al ápice en forma asimétrica, con el extremo curvado. Se distribuyen densa e irregularmente, presentan diferentes tamaños. En C. speciosa, los aguijones son típicamente cónicos y simétricos, generalmente de ápice recto y muy agudo. Se disponen de forma semidensa a esparcida con regular distribución y tamaño. Fig. 1, C-D.

\section{Nomófilos}

El margen de los folíolos en los nomófilos juveniles y adultos es una de las diferencias más destacadas al estado vegetativo, que puede ser irregularmente aserrado hasta entero (raro) en aproximadamente la mitad o tercio apical de la lámina en $C$. chodatii; en tanto que en $C$. speciosa es regular y casi completamente aserrado (excepto en $0,5-1,5 \mathrm{~cm}$ basal). Los dientes también son diferentes, generalmente curvos en $C$. chodatii y rectos en $C$. speciosa. La nerviación en $C$. chodatii es semicraspedódroma (Moreno, 1984), irregular, con 12-15 nervios secundarios completos, distanciados 4-10 mm. En C. speciosa es caspedódroma mixta (Moreno, 1984), regularmente paralela y con nervios completos más numerosos (18-20) distanciados 2-3 $\mathrm{mm}$. Lo mismo se reproduce en los nomófilos de las plántulas. Fig. 1, E-F, G-H.

\section{Flores}

Corola. En C. chodatii la forma de la corola es infundibuliforme con pétalos erectos y el tercio apical reflexo y curvado; en $C$. speciosa la corola es rotácea o estrellada con pétalos abiertos y recurvados desde la base, exponiendo el extremo de la columna de estaminodios. En cuanto a color los pétalos en C. chodatii son blanco cremosos (Digilio \& Legname, 1966) en la lámina y amarillos o marrones en la uña del haz (mitad basal), a menudo con algunas estrías longitudinales purpúreas irregularmente distribuidas. En C. speciosa el color de la lámina es magenta oscuro o rosado con uña blanca o amarilla y abundantes estrías oscuras en la mitad de la lámina. Respecto al margen de los pétalos, es liso en toda la lámina o ligeramente ondulado en el tercio apical en $C$. chodatii; en tanto que en $C$. speciosa es totalmente ondulado incluida la uña. La uña es más ancha en $C$. chodatii, de 1,5$1,6 \mathrm{~cm}$ en el extremo superior y de $0,9-1 \mathrm{~cm}$ en el extremo inferior; en $C$. speciosa es de 0,7-0,9 cm extremo superior y $0,5-0,7 \mathrm{~cm}$ en el inferior. La pubescencia en haz y envés en ambas especies es semejante. Fig. 1, I-L, M, N.

Androceo. Columna de estaminodios de color amarillo homogéneo en toda su longitud en $C$. chodatii con 10 lóbulos apareados y divergentes, generalmente glabros, a veces con escasos pelos blancos o amarillos breves (hasta 3-4 células); lóbulos de 0,15-0,3 cm long. En C. speciosa la columna de estaminodios es blanquecina con 10 lóbulos simétricamente distribuidos, rosados a rojo oscuros, densamente cubiertos por tricomas pluricelulares largos y blancos; lóbulos de 0,3- 
Bol. Soc. Argent. Bot. 53 (2) 2018
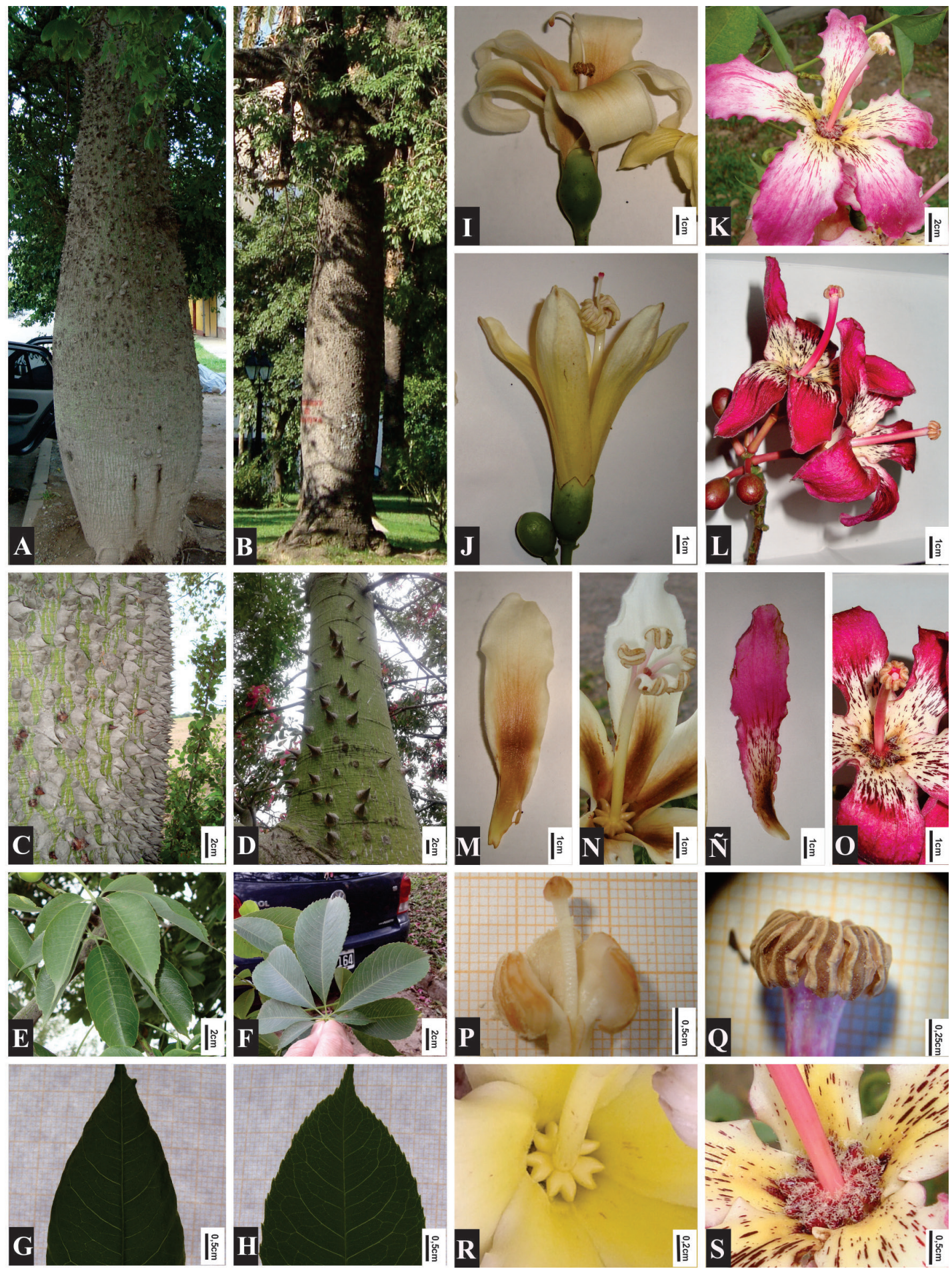

Fig. 1. Ceiba chodatii . A: tronco. C: aguijones. E: hoja. G: folíolo. I-J: flor. M: pétalo. N: androceo. P: collar anteras. R: disco de estaminodios. C. speciosa. B: tronco. D: aguijones. F: hoja. H: folíolo. K-L: flor. $\tilde{\mathbf{N}}$ : pétalo. O: androceo. Q: collar anteras. S: disco de estaminodios. 
0,7 cm long. Columna estaminal amarilla en $C$. chodatii; a menudo con los filamentos libres en el ápice unos 0,25-3,5 cm long.; cuando unidos, el collar de anteras de $0,8 \times 1,3-1,5 \mathrm{~cm}$; anteras de 0,5-0,8 $\times 0,2-0,4 \mathrm{~cm}$. En C. speciosa la columna es rosado clara y los filamentos siempre se encuentran totalmente concrescentes hasta el ápice; también hay concrescencia en el collar de anteras, éste de 0,4-0,5 × 0,6-0,9 cm; anteras de 0,4-0,5 × 0,12-0,2 cm. Fig. 1, N, O-S.

Gineceo. Con forma de cono truncado en $C$. chodatii, con tricomas apicales cortos y gruesos; la base del estilo con tricomas estrellados con 2-8 ramas desiguales de $94-223 \times 17 \mu \mathrm{m}$ y extremos aguzados; también tricomas simples de hasta $168 \times 35 \mu \mathrm{m}(\mathrm{MEB})$; estigma de 2,30 × 1,82 mm (MEB), blanquecino, ovoide, con papilas largas, densas, irregulares, cónicas, de ápice largo delgado y curvado, de 155-260 × 43-49 $\mu \mathrm{m}$ (MEB). En $C$. speciosa, gineceo conoidal con extremo prolongado en el estilo, ápice glabro, base del estilo con tricomas simples, rectos, muy agudos, de $160 \times$ $28 \mu \mathrm{m}$ (MEB); estigma esférico a obovoide, de 1,66 × 1,92 mm (MEB), rosado claro, afelpado con papilas cortas cónicas con extremo redondeado y ligeramente curvado de $83 \times 22 \mu \mathrm{m}$ (MEB). Fig. 2, A-H.

Fruto. La cápsula con cinco valvas, adopta indistintamente tamaños y formas muy variables en ambas especies, desde cilíndricas largas, cortas, ovoides, elipsoides o piriformes con extremos redondeados y/o aguzados. La única diferencia interespecífica es el espesor de las valvas (epi y mesocarpo), notablemente más gruesas en $C$. speciosa, de 6,17-14 $\mathrm{mm}$ y de 2-6,11 $\mathrm{mm}$ en $C$. chodatii. Fig. 2, I-J, O, Q.

Semilla. En ambas especies son semejantes en tamaño y coloración; en general más largas que anchas. Los promedios de todas las dimensiones en 100 semillas muestran mínimas diferencias y los rangos (mínimo y máximo) son iguales. En $C$. chodatii las dimensiones promedio son: 7,78 $\times 6,21$ $\times 4,65 \mathrm{~mm}$; en C. speciosa de 7,79 × 6,11 × 4,59 $\mathrm{mm}$. La testa es castaña a negruzca en $C$. chodatii y castaña en $C$. speciosa, regularmente mamelonada en ambas especies. En lupa binocular y al MEB se observa en $C$. chodatii que los mamelones presentan depresiones circulares centrales, con emergencia de 1-2 tricomas blancos, simples, largos y cortos. En C. speciosa los mamelones se observan sin depresiones y sin tricomas. Fig. 2, K-L, M-N.

Desarrollo de plántulas. En una experiencia realizada en diciembre de 2016, se colocaron en macetas 80 semillas germinadas de $C$. chodatii y 80 de $C$. speciosa, colectadas en julio del mismo año, en cámara de germinación a $28^{\circ} \mathrm{C}$ y fotoperíodo $12 / 12$ h. A las $48 \mathrm{~h}$ emergieron 24 plántulas de chodatii (24\%) y 7 de speciosa $(8,75 \%)$, posteriormente no hubo más emergencia. A los 5 días, el hipocótilo de 2-3 cm crecía curvado en $C$. chodatii con los cotiledones cubiertos por la testa, en tanto que en speciosa el hipocótilo de $2,5 \mathrm{~cm}$ crecía erguido y los cotiledones se encontraban plegados o cubiertos por la testa. A los 15 días, las plántulas se diferenciaron en tamaño de hipocótilo y de cotiledones, desarrollo de protófilo y epicótilo. A los 30 días, en $C$. chodatii el desarrollo del hipocótilo era notoriamente mayor entre 2-4 cm long. con respecto a C. speciosa y también los cotiledones la superaban en 1-2 cm en largo y ancho, el epicótilo en aproximadamente $1 \mathrm{~cm}$ y el protófilo en 0,2-0,3 cm.; además de un nomófilo bien desarrollado, que en $C$. speciosa iniciaba recién su desarrollo. Tabla 1, Fig. 2, N, P.

\section{Tratamiento Taxonómico}

1. Ceiba chodatii (Hassl.) Ravenna, Onira 3(15): 44. 1998. Chorisia chodatii Hassl., Bull. Herb. Boissier ser. 2, 7: 174. 1907. Tipo: Paraguay, Chaco septentrional, en campo Santa Elisa, 1903, Rojas s. n. in herb. Hassler 2849 (Lectotipo G!, designado por Gibbs \& Semir, Ann. J. Bot. Madrid 60(2): 274. 2003).

Fenología. Florece desde febrero hasta mayo en Salta y Jujuy (obs. pers.); desde enero a julio en Tucumán (Digilio \& Legname, 1966). Frutos bien desarrollados, no dehiscentes, desde fines de mayo hasta mediados de setiembre (obs. pers.) y con maduración posterior hasta diciembre (Erise, 1997). Se observó en el área de estudio que la floración no es regularmente anual en todas las poblaciones y también a menudo a pesar de una abundante floración no se producen frutos; por lo que puede considerarse una especie vecera. 
Bol. Soc. Argent. Bot. 53 (2) 2018
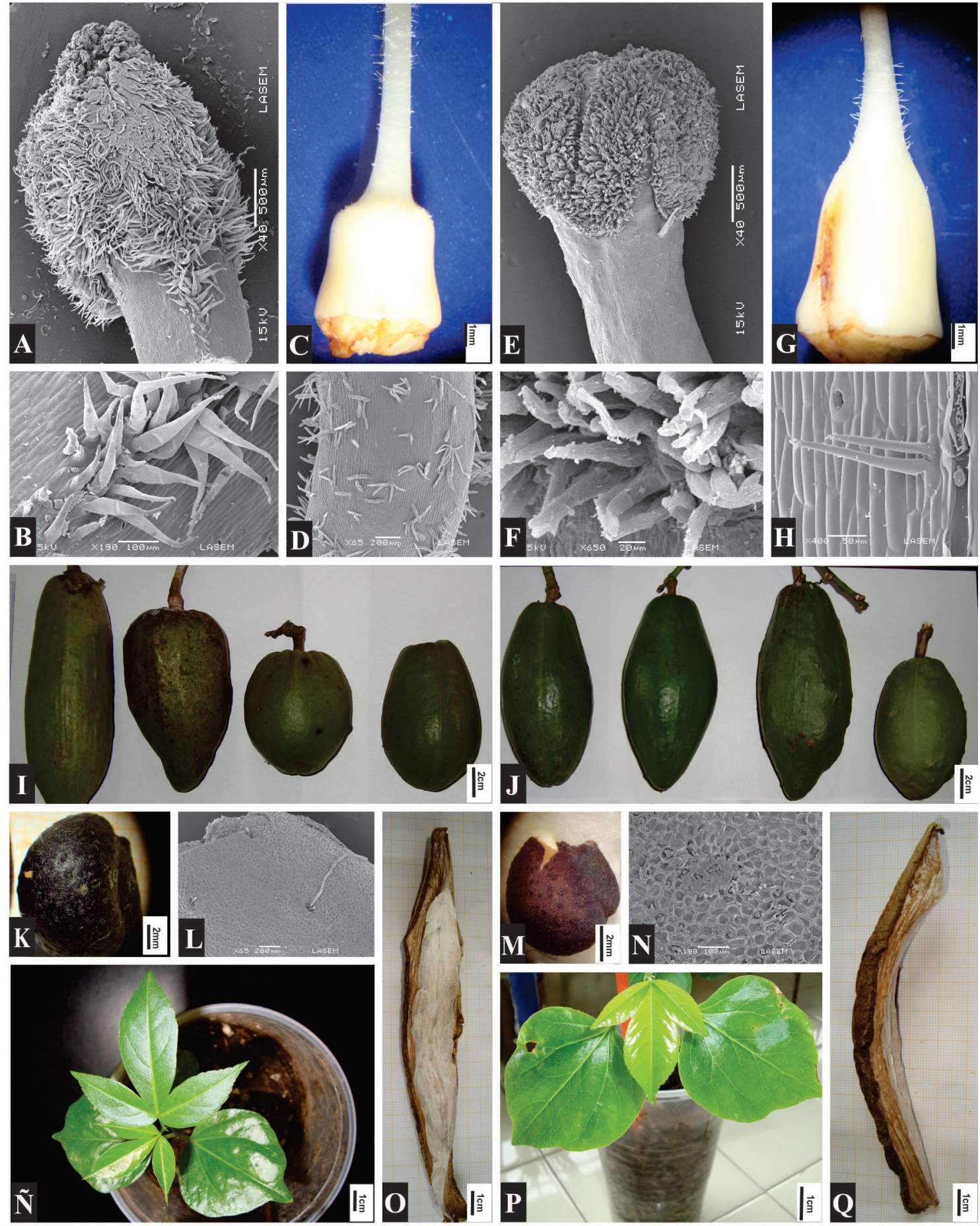

Fig. 2. Ceiba chodatii. A-B: estigma y tricomas del estigma. C-D: gineceo y tricomas del estilo. I: fruto. O: valva del fruto. K-L: semilla (lupa) y episperma (MEB). Ñ: plántula de 20 días. C. speciosa. E-F: estigma y tricomas del estigma. G-H: gineceo y tricomas del estilo. J: fruto. Q: valva del fruto. $\mathbf{M}-\mathbf{N}$ : semilla en proceso germinativo (lupa) y episperma (MEB). P: plántula de 20 días. 


\section{E. C. Lozano y M. A. Zapater - Delimitación taxonómica en Ceiba}

Tabla 1. Ceiba chodatii y C. speciosa. Desarrollo de plántulas durante 30 días.

\begin{tabular}{|c|c|c|}
\hline Órgano & C. chodatii & C. speciosa \\
\hline \multicolumn{3}{|c|}{5 días } \\
\hline Hipocótilo & 2-3 cm curvado & $2-2,5 \mathrm{~cm}$ erguido \\
\hline Cotiledones & Plegados, generalmente cubiertos por la testa & Plegados o cubiertos por la testa \\
\hline \multicolumn{3}{|c|}{15 días } \\
\hline Hipocótilo & 9-10 cm long. & 4-6 cm long. \\
\hline Pecíolo del cotiledón & 0,7-1,3 cm long. & $0,3-0,4 \mathrm{~cm}$ long. \\
\hline Cotiledones & $5-6,5 \times 4,8-5,4 \mathrm{~cm}$ & $2,5-4 \times 2,2-3,9 \mathrm{~cm}$ \\
\hline Epicótilo & $0,5-1 \mathrm{~cm}$ & - \\
\hline Pecíolo del protófilo & 0,3-,0,5 cm long. & - \\
\hline Protófilo (folíolo más largo) & $1-3,2 \times 0,3-1,8 \mathrm{~cm}$ & - \\
\hline \multicolumn{3}{|c|}{30 días } \\
\hline Hipocótilo & $12,3-14,3 \mathrm{~cm}$ long. & $8,5-10 \mathrm{~cm}$ long. \\
\hline Pecíolo del cotiledón & 1,6-1,7 cm long. & $1,1-1,8 \mathrm{~cm}$ long. \\
\hline Cotiledones & $7-7,2 \times 6,4-6,5 \mathrm{~cm}$ & $5,2-6 \times 4,2-4,4 \mathrm{~cm}$ \\
\hline Epicótilo & $3-3,9 \mathrm{~cm}$ & $1,6-3 \mathrm{~cm}$ \\
\hline Pecíolo del protófilo & $2,5-3,5 \mathrm{~cm}$ long. & $1,8-2,4 \mathrm{~cm}$ \\
\hline Protófilo (folíolo más largo) & $6-6,8 \times 2,9-3,3 \mathrm{~cm}$ & $5,7-6,5 \times 2,7-3 \mathrm{~cm}$ \\
\hline Pecíolo de eófilo & $1-3,8 \mathrm{~cm}$ & - \\
\hline Eófilo ( folíolo más largo) & $6,4-8 \times 2,9-3,9 \mathrm{~cm}$ & - \\
\hline
\end{tabular}

Distribución geográfica y hábitat. Ravenna (1998) cita para la especie una distribución muy amplia en la región Chaqueña de Paraguay, norte y noreste de Argentina (Santa Fe, Chaco y Formosa); también en el noroeste (Salta, Tucumán, Santiago del Estero, Catamarca y La Rioja). Esta podría no ser la real distribución geográfica de las poblaciones naturales. Más adecuada sería la distribución aportada por Gibbs \& Semir (2003), según la cual C. chodatii habita en los bosques estacionales secos de Paraguay, Bolivia y piedemonte del oeste de Argentina (Jujuy, Salta, Tucumán, Catamarca y La Rioja); también en algunas otras áreas chaqueñas de Santiago del Estero, Formosa y Chaco. Por otra parte, esta especie al encontrarse ampliamente cultivada también genera confusiones en su distribución.
En el marco de este estudio se comprobó con nuestro relevamiento y trabajo de colección la existencia de un área de distribución natural a lo largo del piedemonte de Yungas (Selva Pedemontana y Ecotono con Chaco Occidental) y en el Chaco serrano del noroeste de Argentina en las provincias de Jujuy, Salta y Tucumán. En esta zona, la distribución se muestra discontinua con presencia de poblaciones concentradas en algunas áreas serranas, probablemente con hábitat asociado a sustratos con altos contenidos de calcio con base en el análisis de los suelos de la distribución (Nadir \& Chafatinos, 1990), lo que debería ser comprobado mediante estudios específicos.

Material estudiado. ARGENTINA. Prov. Catamarca: Dpto. Capital, en "La Quebrada" en 
el cerro, 15-II-1945, Krapovickas 17920 (LIL). Dpto. Santa Rosa, Alijilan, 26-I-1942, Pierotti 11549 (LIL). Prov. Chaco: Dpto. Rio Bermejo, Las Palmas, 15-III-1947, Malvarez 1299 (LIL). Prov. Jujuy: Dpto. El Carmen: Ruta 34 pasando caminera Salta - Jujuy, sobre ruta vieja, 28-II-2014, Zapater \& Lozano 4330 (MCNS). Dpto. Ledesma, Yuto en bosque de EECT - INTA, 21-II-2014, Zapater \& Lozano 4320 (MCNS). Prov. Salta: Depto. Capital, Salta en camino al Santuario de la Virgen del Cerro, Co 20 de Febrero, 21-II-2015, Zapater \& Lozano 4550 (MCNS). Dpto. Gral. Güemes, Autopista a Salta, $5 \mathrm{~km}$ de entrada a TermoAndes, 28-II-2014, Zapater \& Lozano 4335 (MCNS); a 1 km de entrada a Cobos sobre Autopista Aunor, Ruta 9, 14-III2014, Zapater \& Lozano 4339 (MCNS). Dpto. Gral. San Martín, Ruta 81, 8 km al W de Dragones, 9-IV-1996, Krapovickas y Cristóbal 46718 (LIL); Campamento Tablillas, 3-7 km al W de Gral. Mosconi, 1-II-1997, Palma 196 (MCNS); Tartagal, sobre ruta nacional 34, 19-II-2000, Tolaba 2650 (MCNS). Dpto. La Viña: Coronel Moldes, Ruta 68 $\mathrm{km} \mathrm{123,} \mathrm{en} \mathrm{lomada} \mathrm{a} 1 \mathrm{~km}$ al W del camino, 1-III1989, Novara et al. 8633 (MCNS). Dpto. Metán: Río Piedras, Ruta 34, 12-IX-2014, Zapater \& Lozano 4501 (MCNS); Lumbreras, Ruta 34 a 100 m de la Planta Distribuidora de Gas, al costado de la Ruta, 29-II-2015, Zapater \& Lozano 4552 (MCNS). Dpto. Orán, Arroyo Bananal, 28-III-1972, Meyer y Vaca 23558 (LIL). Dpto. Rosario de Lerma, Campo Quijano, a orilla de canal de riego, 3-III-2014, Zapater \& Lozano 4336 (MCNS). Prov. Santa Fe: Dpto. Rosario, Costanera frente al Monumento La Bandera, 15-I-2015, Zapater \& Lozano 4550 (MCNS). Prov. Tucumán: Dpto. Capital, Barranca colorada, 24-IV-1925, Venturi 167 (LIL). Dpto. Burruyacu, Macomita, 10-III.1944, Varela s.n. (LIL). Dpto. Trancas, Ruta 34, a 3 km de Vipos hacia San Miguel, en banquina, 24-II-2014, Zapater \& Lozano 4325 (MCNS).

Observaciones. El estudio del lectotipo designado por Gibbs y Semir (2003) mediante JSTOR Plant Science, nos muestra discordancias en las dimensiones florales respecto a las que constan en la descripción de Ravenna (1998) para la nueva combinación de esta especie y con nuestros registros, entre otros la longitud de las anteras de 4,4 mm según Ravenna y de 5-8 mm long. en el material estudiado. También se aprecian diferencias en algunos caracteres no cuantitativos como la pubescencia que Ravenna menciona para los estaminodios los que observamos a menudo glabros y solo a veces con tricomas cortos esparcidos; como también la eventual pigmentación rojiza del extremo de los estaminodios, que no se encuentra en el material estudiado. El lectotipo, aunque cuenta con 4 flores completas, no presenta el tubo estaminal dividido en ninguna de ellas y las anteras se encuentran separadas solo en un material suelto de sobre, a pesar de que generalmente la columna estaminal está apicalmente dividida y también el collar de anteras en los ejemplares nativos del área en estudio (solo raramente la columna se encuentra entera y el collar de anteras indiviso). Lo que si resulta completamente concordante entre el lectotipo y los ejemplares que estudiamos son el diámetro del collar de anteras y la longitud de las anteras. Respecto a las hojas del ejemplar, el aserramiento de la mitad superior de los márgenes es claramente coincidente con el material estudiado de $C$. chodatii, carácter de diferenciación con $C$. speciosa.

A campo, las flores se observaron a menudo atacadas por insectos de gran tamaño (coleópteros) que destruyen la base de la flor y afectan al gineceo.

2. Ceiba speciosa (A. St.-Hil.) Ravenna, Onira 3: 46. 1998. Chorisia speciosa A. St.-Hil. in A. St.Hil., A. Juss. \& Cambess., Pl. Usuel. Bras., tab. 63. 1827. Tipo: Brasil, Provincia de Minas Gerais, Saint Hilaire s.n., (Lectotipo P!, designado por Gibbs \& Semir, Ann. J. Bot. Madrid 60(2): 271. 2003).

Fenología. Floración de enero a mayo. Frutos bien desarrollados, no dehiscentes, desde fines de mayo hasta mediados de setiembre, coincidente con $C$. chodatii en estado de cultivo en el área de estudio. Los frutos se encuentran maduros desde setiembre hasta diciembre.

Distribución geográfica y hábitat. Citado por Ravenna (1998) para Brasil desde el SE de Minas Gerais y a través de los estados de Río de Janeiro, San Pablo, Paraná y Santa Catarina, hasta E - SE de Paraguay y NE de Argentina, en los bosques 1luviosos. Según Gibbs \& Semir (2003) habita en el noreste, sudeste y oeste de Brasil, norte de Argentina, Paraguay, Bolivia, sur y centro de Perú. En Argentina vive en Misiones, Corrientes, Chaco 


\section{E. C. Lozano y M. A. Zapater - Delimitación taxonómica en Ceiba}

y Formosa (Selva Misionera y Parque Chaqueño Oriental) (Tortorelli, 2009). Mencionado como un árbol del dosel y emergente en los bosques semi-siempreverdes hasta deciduos, en suelos bien drenados y también en bosque húmedo de valles (Jardim et al., 2003).

Material estudiado. ARGENTINA. Prov. Corrientes: Dpto. Santo Tomé, a $35 \mathrm{~km}$ al SW de Santo Tomé, A ${ }^{\circ}$ Cuay Grande, 5-II-1979, Schinini et al. 16751 (LIL). Prov. Formosa: Dpto. Pilagá, Misión Tacahaglé, 11-XI-1949, Morel 8870 (LIL). Prov. Jujuy: Dpto. Palpalá, entrada a ciudad de Palpalá en autopista, cultivado, 28-II-2014, Zapater \& Lozano 4326 (MCNS). Prov. Misiones: Dpto. Candelaria, Santa Ana, 20-I-1913, Lillo 12348 (LIL). Dpto. Eldorado, Eldorado, 29-III-1945, Bertoni 1087 (LIL). Dpto. Iguazú, Puerto Aguirre, 13-II-1945, Hayward s.n. (LIL); Col. Victoria, 3-IV-1945, Bertoni 107 (LIL). Dpto. Libertador Gral. San Martín, Puerto León, 26-VI-1909, Venturi 70 (LIL). Dpto. San Javier, Tres Bocas, 12-II-1947, Schwarz 4107 (LIL). Prov. Salta: Dpto. Capital, $\mathrm{B}^{\mathrm{o}} 20$ de Febrero, Calle Alsina al costado de vías ferrocarril entre Alvear y Bolívar, 20-II-2014, Zapater \& Lozano 4296 (MCNS); Plaza 9 de Julio, cultivado, 20-II-2014, Zapater \& Lozano 4300 (MCNS); Monumento a Güemes, cultivado, 20-II-2014, Zapater \& Lozano 4303 (MCNS); Tres Cerritos, Calle Los Saucos y Las Higueras, cultivado en vereda, 20-II-2014, Zapater \& Lozano 4304 (MCNS); $2^{\circ}$ Rotonda Tres Cerritos, cultivado, 20-II-2014, Zapater \& Lozano 4305 (MCNS). Dpto. Güemes, ciudad de Güemes, en calle paralela a Ruta 34 al ingreso a la ciudad frente Estación de Servicio, 28-II-2014, Zapater \& Lozano 4333 (MCNS). Prov. Santa Fe: Dpto. Rosario, Monumento a la Bandera, cultivado, 15I-2015, Zapater \& Lozano 4545 (MCNS).

Material adicional estudiado (ejemplares singulares o potenciales híbridos). Prov. Santa Fe: Dpto. Rosario, sobre Costanera, cerca Monumento a La Bandera (cultivado),15-I-2015, Zapater \& Lozano 4546 (MCNS); ibid., Zapater \& Lozano 4547 (MCNS); en Monumento a La Bandera, cultivado, corola rosado clara, 15-I-2015, Zapater \& Lozano 4548 (MCNS); ibid., corola rosado clara, Zapater \& Lozano 4549 (MCNS).
Observaciones. El lectotipo de C. speciosa estudiado mediante JSTOR Plant Science, muestra un ejemplar con todos los folíolos elípticos con margen completamente aserrado y numerosos nervios paralelos aproximados, lo que concuerda con los caracteres de diferenciación en márgenes y nerviación con $C$. chodatii. Respecto a la forma de los folíolos, en los ejemplares estudiados es frecuentemente variable en la misma hoja, siendo en la mayoría elípticos los basales y obovados los laterales y centrales. En cuanto a la única flor del ejemplar, si bien la forma estrellada o rotácea de la corola no se encuentra expresada, hay coincidencia en el borde totalmente ondulado de los pétalos; también en las dimensiones del collar de anteras y la longitud de las anteras, por lo que podemos afirmar que los ejemplares cultivados y nativos que estudiamos corresponden a C. speciosa. En su monografía de Ceiba, Ravenna (1998), no describe a $C$. speciosa y solo marca diferencias con $C$. insignis que presenta posteriormente en una clave, por lo cual no se pueden corroborar características descriptivas de esta especie, pero al menos un carácter destacado por el autor como la longitud de las anteras, de 7-8 $\mathrm{mm}$, no es coincidente con nuestros registros de $4-5 \mathrm{~mm}$ long.

Se observó una importante variación en la pigmentación de la corola desde rosado pálido hasta magenta (visto en ejemplares cultivados). Ejemplares coleccionados en Rosario (Santa Fe) en el Monumento a la Bandera y adyacencias sobre la Costanera, (Zapater \& Lozano 4546, 4547, 4548 y 4549 MCNS), presentaron la columna de estaminodios con escasos tricomas cortos o largos, lóbulos de 0,2-0,3 cm long.; el tubo estaminal unido o dividido apicalmente $1,2-1,8 \mathrm{~cm}$, erecto o resurpinado como en C. pubiflora y anteras de 0,7 $0,75 \times 0,25-0,3 \mathrm{~cm}$ unidas o separadas, caracteres coincidentes con $C$. chodatii. Sin embargo, también presentan caracteres vegetativos de C. speciosa como el tipo de margen y nerviación de los folíolos, sumado al color rosado de la flor. Estos ejemplares al presentar caracteres de ambas especies podrían validar un caso de hibridación como mencionan Gibbs \& Semir (2003).

La especie florece abundantemente y fructifica todos los años en los ejemplares cultivados en el área de estudio, a diferencia de $C$. chodatii. Cabría confirmar si esto se sostiene en su distribución natural. 


\section{Clave para diferenciar C. chodatii de C. speciosa sobre la base de caracteres florales}

1. Corola infundibuliforme con pétalos erectos y deflexos en la mitad superior, color crema, a veces con algunas estrías rojizas, margen liso o apenas ondulado en 1/3 apical. Columna de estaminodios amarilla, lóbulos de 0,15-0,3 cm long. apareados y divergentes, generalmente glabros. Columna estaminal a menudo con filamentos libres en el ápice; anteras de 0,5-0,8 × 0,2-0,4 cm. Gineceo en forma de cono truncado. C. chodatii

1 '. Corola estrellada a rotácea, pétalos abiertos rosados o magenta con base amarilla y con abundantes estrías oscuras, margen completamente ondulado. Columna de estaminodios blanquecina, lóbulos rosados o rojo oscuros de 0,3-0,7 cm long., completamente simétricos, densamente cubiertos por largos tricomas. Columna estaminal con filamentos siempre concrescentes hasta el ápice; anteras concrescentes de 0,4-0,5 × 0,12-0,2 cm. Gineceo conoidal, extremo prolongado con el estilo. C. speciosa

\section{Clave para diferenciar C. chodatii de $C$. speciosa sobre la base de caracteres vegetativos $y$ de fruto}

1. Tronco con aguijones asimétricos de ápice curvado. Folíolos con margen irregularmente aserrado en $1 / 2$ a 1/3 apical o entero, dientes curvos; nerviación semicaspedódroma; 12-15 nervios secundarios completos. Valvas del fruto de 2-6 $\mathrm{mm}$ de espesor. Semilla con mamelones con depresión apical y 1-2 tricomas simples. C. chodatii

1'. Tronco con aguijones simétricos de ápice recto y muy agudo. Folíolos con margen regularmente aserrado casi hasta la base, dientes rectos; nerviación caspedódroma mixta; 18-20 nervios secundarios completos. Valvas del fruto de 6,2$14 \mathrm{~mm}$ de espesor. Semillas con mamelones sin depresiones ni tricomas. C. speciosa

\section{Discusıón}

Una de las diferencias marcadas por Gibbs \& Semir (2003) entre las dos especies estudiadas se refiere a la pubescencia de los lóbulos de los apéndices estaminales, mencionados como glabros en $C$. chodatii y usualmente pilosos en $C$. speciosa. Se ha observado en $C$. chodatii que estos apéndices pueden ser glabros o poseer tricomas cortos, esparcidos y amarillentos. Por otra parte en $C$. speciosa los lóbulos de los apéndices estaminales son siempre abundantemente pilosos con tricomas largos, flexuosos y blancos.

Si bien Gibbs \& Semir (2003) mencionan que ocasionalmente ejemplares de $C$. speciosa y de $C$. chodatii pueden presentar el extremo superior del tubo estaminal dividido en 5 filamentos, este carácter es habitual en $C$. chodatii en ejemplares nativos y cultivados a la madurez de la flor. En C. speciosa, el tubo estaminal es completamente concrescente incluidas las anteras hasta la madurez de la flor, carácter observado en todos los ejemplares estudiados; solamente se encontró la columna dividida en los potenciales híbridos de Rosario, prov. de Santa Fe.

Dentro de la clave de las especies del género Ceiba, Gibbs \& Semir (2003), realizan un agrupamiento de especies al que designan como "Ceiba insignis aggregate" sobre la base de los siguientes caracteres: flores con filamentos estaminales unidos que forman un tubo terminado en un collar de anteras, ocasionalmente con alguna fisión terminal del tubo. Dentro de este agrupamiento, C. chodatii y Ch. insignis tienen una problemática historia, según relatan Gibbs y Semir (2003); que Hicken en 1900, identificó los árboles de $C$. chodatii cultivados en La Recoleta en Buenos Aires como Chorisia insignis H.B.K. y como consecuencia este nombre fue ampliamente utilizado para denominar las especies colectadas en Argentina, Bolivia y Perú, como también los ejemplares cultivados, originándose una importante confusión taxonómica con Chorisia insignis Kunth, sinónimo de C. insignis (Kunth) Gibbs \& Semir.

Analizando características citadas por Gibbs \& Semir (2003) para C. chodatii y C. insignis, se encuentran las siguientes diferencias: 1) $C$. chodatii tiene pétalos blanquecinos a amarillos y C. insignis puede poseer pétalos rosado pálidos, no siempre blancos; 2) los estaminodios de $C$. chodatii son blanco amarillentos y los de $C$. insignis tienen colores variables desde blanco hasta rojo anaranjado; 3) el tubo estaminal en $C$. chodatii es blanco cremoso y en $C$. insignis es rosado; 4) los pétalos de $C$. chodatii son espatulados y en $C$. insignis angostamente oblongos; 5) la distribución de C. insignis es el sur de Ecuador y norte de Perú. 
Dentro del agrupamiento $C$. insignis, se encuentran otras dos especies muy afines, C. speciosa y $C$. crispiflora (Kunth) Ravenna, caracterizadas por poseer en común la corola con pétalos magenta en la mitad superior, con estrías rojo oscuras y base amarillenta. Las diferencias entre ambos taxones son: 1) C. speciosa tiene columna de estaminodios glabra, en tanto que en C. crispiflora es serícea; 2) el ancho de los pétalos es mayor a $1,5 \mathrm{~cm}$ lat. en $C$. speciosa, en tanto que en crispiflora es de 0,6-1 cm lat. Estos caracteres delimitan ambas especies.

Tanto $C$. chodatii como $C$. speciosa, no se observaron con afectaciones por heladas en la zona de estudio, a pesar de que Tortorelli (2009) menciona que ambas son sensibles a heladas.

\section{Conclusiones}

Se descarta para Argentina la presencia de $C$. insignis en estado natural por sus características diferenciales con $C$. chodatii y su distribución. También se descarta la presencia como nativa de $C$. crispiflora por lo analizado precedentemente y su distribución en Brasil, Minas Gerais y Rio de Janeiro.

Se mantiene por lo tanto la existencia de las tres especies conocidas de Ceiba para Argentina: $C$. chodatii, C. speciosa, y C. pubiflora, esta última no estudiada en este trabajo, por ser claramente identificable por sus filamentos estaminales libres y resurpinados, además de su particular y reducida área de distribución en Misiones sobre suelos calcáreos.

Para las dos especies objeto de estudio, se agregaron a los iniciales caracteres de diferenciación exclusivamente florales no cuantitativos, unos 20 nuevos caracteres florales, vegetativos, de fruto, semilla y plántula cuali- y cuantitativos, que permitirán una segura determinación de las dos especies.

\section{Bibliografía}

BYNG, J. W., M. W. CHASE, M. J. M. CHRISTENHUSZ, M. F. FAY, W. S. JUDD, D. J. MABBERLEY, A. N. SENNIKOV, D. E. SOLTIS, P. S. SOLTIS \& P. F. STEVENS. 2016. An update of the Angiosperm Phylogeny Group classification for the orders and families of flowering plants: APG IV. Bot. J. Linn. Soc., pp. 108.
ANTON, A. M. \& F. O. ZULOAGA. 2017. Bombacaceae. In: ANTON, A. M. \& F. O. Zuloaga (eds.), Flora Argentina. http://www.floraargentina. edu.ar

BAUM, D. A., W. S. ALVERSON \& R. NYFFELER. 1998. A durian by any other name: taxonomy and nomenclature of the core Malvales. Harv. Pap. Bot. 3: 315-330.

BAUM, D. A., S. D. SMITH, A. YEN, W. S. ALVERSON, R. NYFFELER, B. A. WHITLOCK \& R. L. OLDHAM. 2004. Phylogenetic relationships of Malvatheca (Bombacoideae and Malvoideae; Malvaceae sensu lato) as inferred from plastid DNA sequences. Am. J. Bot. 91: 1863-1871.

DIGILIO, P. L. \& P. R. LEGNAME 1966. Los árboles indígenas de la provincia de Tucumán. Opera Lilloana 15: 76.

ERIZE, R. 1997. El Nuevo Libro del Árbol 1-2. Ed. El Ateneo, Buenos Aires.

GIBBS, P. E., J. SEMIR \& N. D. DA CRUZ 1988. A proposal to unite the genera Chorisia Kunth with Ceiba Miller (Bombacaceae). Notes Roy. Bot. Gard. Edinburgh 45: 125-136.

GIBBS, P. \& J. SEMIR. 2003. A taxonomic revision of the genus Ceiba Mill. (Bombacaceae). Anales Jard. Bot. Madrid 60: 259-300.

JARDIM, A., T. J. KILLEN \& A. FUENTES. 2003. Guía de los árboles y arbustos del Bosque Seco Chiquitano, Bolivia,pp.324. Editorial FAN, Santa Cruz de la Sierra, Bolivia.

KUNTH, C. 1822. Chorisia. In: F. Humbolt, A. Bonpland \& C. Kunth (eds.), Nova genera et species plantarum 5: 285-298. Paris.

MORENO, N. P. 1984. Glosario botánico ilustrado, pp. 300. Compañía Editorial Continental S. A., México.

NADIR, A. \& T. CHAFATINOS. 1990. Los Suelos del N.O.A. Tomo 1, 2, 3 (Salta y Jujuy).INTA Salta, Argentina.

NOVARA, J. L. 1993. Bombacaeae Kunth. In: NOVARA, J. L. (ed.), Flora del Valle de Lerma 1(20): 1-8. Edición digital 2012.

PRADO, D. E. \& P. E. GIBBS 1993. Patterns of species distributions in the dry seasonal forests of South America. Ann. Missouri Bot. Gard. 80: 902-927.

RAVENNA, P. 1998. On the identity, validity, and actual placement in Ceiba of several Chorisia species (Bombacaceae), and description of two new South American species. Onira 3: 42-51

SCHUMANN, K. M. 1886. Bombacaceae. In: MARTIUS, C. F. (ed.), Fl. Bras. 12(3): 201-250. München, Wien, Leipzig.

SCHUMANN, K. M. 1890. Bombacaceae. In: ENGLER, A. \& K. Prantl (eds), Nat. Pflanzenfam. 3(6): 53-68. Leipzig. 
Bol. Soc. Argent. Bot. 53 (2) 2018

STEVEnS, W. D., UlloA U., POOL A. \& O. M. MONTIEL 2001. Flora de Nicaragua. Monogr. Syst. Bot. Missouri Bot. Gard. 85: 1-2666

THIERS, B. [permanentemente actualizado, consulta 2017] Index Herbariorum: a global directory of public herbaria and associated staff. New York Botanical Garden's Virtual Herbarium, http:// sweetgum.nybg.org/ih.

TORTORELLI, L. A. 2009. Maderas y Bosques Argentinos II, pp.1111. Orientación Gráfica Editora, $2^{\circ}$ Edición, Buenos Aires.
ZULOAGA, F. O., O. MORRONE \& M. J. BELGRANO. (eds.). 2008. Catálogo de las Plantas Vasculares del Cono Sur. (Argentina, sur de Brasil, Chile, Paraguay y Uruguay). Monogr. Syst. Bot. Missouri Bot. Gard. 107: 1-3348. http://www2.darwin.edu.ar/Proyectos/ Flora Argentina.

Recibido el 28 de noviembre de 2017, aceptado el 6 de abril de 2018. Editor: Massimiliano Dematteis. 